\title{
Insight into Multiple Sclerosis
}

\author{
Kelli Wehman-Tubbs, MD; Steven H. Yale, MD; and Loren A. Rolak, MD
}

Keywords:

Multiple sclerosis;

Interferon type I;

Demyelinating autoimmune diseases, CNS;

Multiple sclerosis/diagnosis;

Central Nervous System/pathology
Reprint Requests:

Steven H. Yale, MD

Department of Internal Medicine

Marshfield Clinic

1000 North Oak Avenue

Marshfield, WI 54449

Telephone: 7I 5-387-5436

Fax: 7| 5-389-3808

Email:yale.steven@mcrf.mfldclin.edu
Received: December 22, 2004

Accepted: January 6, 2005
$\mathrm{M}$

ultiple sclerosis (MS) is characterized by multiple areas of scarring or inflammation (sclerosis) occurring spatially in time and location throughout the central nervous system (CNS). Recent advances in our understanding of MS have paved the way for new therapies directed towards modulating one or more aspects of the immune response. Despite these advances there is a gap in our knowledge regarding the pathophysiology, epidemiology, and mechanism(s) of action of the drugs used to treat this disease.

\section{Current Knowledge of the Pathogenesis of MS}

MS is a cell- and antibody-mediated white matter disease in which myelin and oligodendrocytes are targets of tissue damage. Myelin and its antigenic epitopes are normally protected from immune surveillance by an intact blood brain barrier. The pathogenesis is incompletely understood. Patients who carry the Class II major histocompatibility complex type HLA-DR2 (e.g., northern Europeans, inhabitants of northern climates) are particularly susceptible. In these individuals it is believed that environmental factors (e.g., viral infections, bacterial polysaccharides, superantigens) may expose macrophages and microglial cells to myelin antigens, resulting in the activation and proliferation of $\mathrm{T}$ lymphocytes. ${ }^{1}$ This leads to a series of events including antibody production (e.g., myelin basic protein, myelin oligodendrocyte glycoprotein) and an up- or down-regulation of the immune response, depending on the type of signal that results from the interaction of surface receptors and ligands found on the T cell and antigen presenting cell. The diverse pathologic mechanisms of MS are reflected by variable clinical responses to immunomodulatory agents used in the treatment of different subtypes of this disease.

\section{Definition of the Most Common Presentation for Patients with MS}

There is no predictable pattern of presentation for patients with MS, which often makes diagnosis clinically challenging. Symptoms that occur most commonly in MS include tremor, optic neuritis or double vision, dysarthria, and dizziness from brainstem dysfunction.

Most patients with MS have the relapsing-remitting (RRMS) subtype characterized by the abrupt episodic onset of symptom(s) occurring over hours or days, peaking 
Table 1. Some disorders that resemble multiple sclerosis.

Vascular

Primary central nervous system vasculitis

Stroke and ischemic disease

Vascular malformations of the spinal cord

Infections

Meningovascular syphilis

HIV associated myelopathy

Lyme disease

Neoplastic

Paraneoplastic syndrome

Tumors of the spinal cord

Lymphoma or glioma

Degenerative

Spinocerebellar ataxia

Genetic

X-linked adrenomyeloneuropathy

Autoimmune

Systemic lupus erythematosus and other collagen vascular diseases

Endocrine/metabolic

Vitamin E deficiency

Hypothyroidism

Vitamin B12 deficiency

within several days, improving gradually or incompletely over days to weeks, and often disappearing entirely within 8 weeks. Thus, in cases where an initial diagnosis is suspected, a clinically confirmed disease will likely declare itself within time. More accurate early diagnosis may become even more critical if drugs used early in the disease delay neurologic deficits.

Approximately $20 \%$ of patients with the RRMS subtype will remain clinically stable or nearly stable for two or more decades. ${ }^{2}$ However, within 25 years the majority of untreated patients with RRMS will transform into a secondary progressive phase characterized by a chronic and steady increase of physical symptoms and disability. ${ }^{3}$

The primary progressive subtype of MS (PPMS) is found in approximately $10 \%$ to $15 \%$ of patients. This variant affects both males and females equally. PPMS differs from the RRMS subtype in that it occurs in older individuals, presents with few lesions on magnetic resonance imaging (MRI), exhibits lower levels of inflammatory markers and myelopathic features, and is unresponsive to immunomodulatory agents. ${ }^{4}$
Tests Available to Assist in the Diagnosis of MS

Definitive diagnosis requires the presence of clinical, laboratory (e.g., evoked potentials or cerebrospinal fluid), and radiographic (e.g., MRI) findings. Cerebrospinal fluid and evoked potentials may initially be normal in 30\% to $40 \%$ and $40 \%$ to $50 \%$ of patients, respectively. ${ }^{5}$ The diagnosis can often be suspected at an early stage based on clinical and MRI findings, since an individual with a clinically isolated syndrome and a MRI showing two to three typical lesions has approximately a $90 \%$ risk of developing MS. ${ }^{5}$

In a case in which a single CNS lesion is found on MRI and the patient has a focal neurologic examination further visual, somatosensory, or auditory evoked potentials may be useful in unmasking an occult abnormality. MRI is sensitive but less specific for the diagnosis of MS. MRI typically shows multiple ovoid and confluent areas that are hyperintense on T2 imaging and perpendicular to and surrounding the ventricles. A host of other disorders that have a predilection for brain white matter can mimic MS both clinically and radiographically (Table 1$)$.

Analysis of cerebrospinal fluid is a sensitive and fairly specific adjunct to the diagnosis of MS. The humoral immune response to myelin antigens is reflected by an increase in immunoglobulin $\mathrm{G}$ (IgG) levels and synthesis rate and oligoclonal bands. Lymphocytic pleocytosis is generally mild with $<50$ cells $/ \mathrm{mm}^{3}$. False positive results may be found in other infectious or inflammatory conditions.

\section{Options Available for Treating the Disease and Complications of MS}

There are no standard recommendations for the treatment of MS because it currently is not possible to predict which patients will experience early progression or a benign course. $^{2}$ MS is a heterogeneous disease so treatment decisions should be individualized for each patient. Until recently, parenteral high dose corticosteroids were the only standard treatment option for MS. Corticosteroids were recommended for the treatment of acute disabling attacks because they shorten symptom duration. Over the past 10 years, the Federal Drug Administration (FDA) approved six new disease-modifying drugs for the treatment of MS. These drugs decrease the risk of subsequent relapses or reduce clinical and MRI findings of the disease.

The beta-interferons (e.g., beta-1a, beta-1b) are FDA approved under the trade names of Avonex (Biogen Idex, Cambridge, MA), Betaseron (Berlex, Montville, NJ), and Rebif (Serono, Inc, Rockland, MA). These agents alter the immune response by a number of complex mechanisms including reducing $\mathrm{T}$ cell proliferation and altering cytokines, adhesion molecules, and proteases. These drugs differ based on their frequency of administration. It is unknown whether the small changes in their molecular structure affect their efficacy. Glatiramer acetate (Copaxone, Teva Pharmaceutical Industries Ltd., North Wales, PA) is a synthetic polypeptide that has four 
Table 2. Medication alternatives for the treatment of secondary symptoms in multiple sclerosis

Problem Treatment options

Spasticity

Baclofen (oral or epidurally), tizanidine, benzodiazepines, botulinum toxin

Detrusor-sphincter dyssynergia

$\alpha$-antagonists (e.g., prazosin, doxazosin), double voiding, vibratory stimulation on hypogastrium

Detrusor hyperreflexia

Anticholinergic agents (e.g., oxybutynin, tolterodine)

Constipation

Perdiem*; senna

Poor rectal evacuation

Suppositories (glycerin or ducolax) and mini-enemas (Enemeez ${ }^{*}$ a minidose enema containing liquefied glycerin and docusate), digital rectal stimulation

Sexual dysfunction

Sildenafil, yohimbine, intracavernosal injection with prostaglandin E, hydraulic pumps, penile implants

Fatigue

Modafinil (Provigil $\left.{ }^{*}\right)$, amantadine (Symmetrel $\left.{ }^{*}\right)$, mixed salts of dextroamphetamine (Adderall*), methylphenidate (Ritalin*), pemoline (Cylert*) and antidepressants (fluoxetine and sertraline)

*Perdiem (Rhône-Poulenc Rorer, Collegville, PA); Enemeez (Western Research Laboratories, Phoenix, AZ); Provigil (Cephalon, Inc., West Chester, PA); Symmetrel (Alliance Pharmaceuticals Ltd, Wiltshire, England); Adderall (Shire Biochem Inc., Quebec, Canada); Ritalin (Novartis Pharmaceuticals Corp., East Hanover, NJ); Cylert (Abbott Laboratories, Abbott Park, IL); Fosamax (Merck \& Co., Inc., Whitehouse Station, NJ); Actonel (Procter \& Gamble Pharmaceuticals, Cincinnati, $\mathrm{OH}$ ).

amino acid sequences antigenically similar to myelin basic protein. ${ }^{6}$ The mechanism of action of Copaxone is also incompletely understood, but seems to interfere with antigen specific $\mathrm{T}$ cell activation. All these drugs reduce the frequency of recurrent episodes, but have little effect on the most disabling secondary progressive phase of the disease. There are currently no well-controlled published clinical studies that have directly compared the interferons or glatiramer acetate in terms of treatment efficacy. Currently treatment decisions are based on convenience of dosing, route of administration (e.g., subcutaneously [Rebif, Betaseron] or intramuscularly [Avonex]), side effects and, exclusive to the interferons, the development of neutralizing antibodies that may decrease their effectiveness. Mitoxantrone (Novantrone, Serono, Inc.) is a chemotherapeutic drug that may be useful in delaying disability in the secondary, rapidly progressive subtype of MS, or in patients with multiple clinically significant relapses. Nataluzimab (Tysabri, Elan Pharmaceuticals, San Diego, CA) is a monoclonal antibody that prevents entry of $\mathrm{T}$ cells across the blood-brain barrier into the CNS and it has recently been approved also to reduce relapses of MS.

A host of other medications are available for the treatment of the associated symptoms of MS (Table 2). When choosing a medication, the potential side effect profile should be considered. Non-pharmacological treatment is also important in improving the quality of life for patients with MS. This may include, among others, single prong straight canes or rolling walkers for patients with gait disorders and physical therapy to maintain functional mobility.

\section{References}

1. Noseworthy JH, Lucchinetti C, Rodriguez M, Weinshenker BG. Multiple sclerosis. N Engl J Med 2000;343:938-952.

2. Wingerchuk DM, Carter JL. Practical consultations: multiple sclerosis. Semin Neurol 2003;23:253-264.

3. Weinshenker BG, Bass B, Rice GP, Noseworthy J, Carriere W, Baskerville J, Ebers GC. The natural history of multiple sclerosis: a geographically based study. I. Clinical course and disability. Brain 1989;112:133-146.

4. Frohman EM. Multiple sclerosis. Med Clin North Am 2003;87:867-897.

5. Hawker K, Frohman E. Multiple sclerosis. Prim Care 2004;31:201-226.

6. Rolak LA. Multiple sclerosis: it's not the disease you thought it was. Clinical Medicine \& Research 2003;1:57-60.

\section{Author Affiliations}

Kelli Wehman-Tubbs, MD, Department of Internal Medicine, Marshfield Clinic, 1000 N. Oak Avenue, Marshfield, Wisconsin 54449. 
Steven H. Yale, MD, Department of Internal Medicine,

Marshfield Clinic, and Clinical Research Center, Marshfield

Clinic Research Foundation, 1000 N. Oak Avenue,

Marshfield, Wisconsin 54449.

Loren A. Rolak, MD, Department of Neurology, Marshfield

Clinic, 1000 N. Oak Avenue, Marshfield, Wisconsin 54449. 